\title{
Indigenous Psychology as a Remedy for the Dualism and Desacralization of Modern Western Psychology
}

\author{
Samuel Bendeck Sotillos ${ }^{1}$ \\ Institute of Traditional Psychology
}

${ }^{1}$ Mental Health Therapist, Writer, and Researcher. E-mail: samuelbendeck@yahoo.com

Healing the Soul Wound: Trauma-Informed Counseling for Indigenous Communities, Second Edition By Eduardo Duran (Tiospaye Ta Woapiye Wicasa), Foreword by Allen E. Ivey New York, NY: Teachers College Press, 2019, PP. 208

Corresponding author: Samuel Bendeck Sotillos E-mail:

samuelbendeck@yahoo. com

eISSN: 2458-9675

Received: 05.08.2021

Revision: 10.08.2021

Accepted: 12.08.2021

CCopyright 2021

by Author(s)
As if out of nowhere psychological trauma has become a commonly used term in everyday conversation. The fact that trauma on a collective level is so widely discussed today is itself a disclosure of not only vulnerability, but the precarious state, if not, spiritual crisis, of the modern world. It beckons the question, is there something triggering about the modern world itself that is creating these conditions? Or is it just a matter of a heightened awareness of trauma and historical trauma, known as transgenerational trauma or intergenerational trauma.

There appears to be a deeper or underlying dimension pertaining to the mass traumatization of the present day that goes unnoticed, which is the trauma due to the loss of the sense of the sacred, what could be called the traumatization of secularism. The vacuum that has been created in the modern world due to the loss religion is not something that can be taken lightly, yet it is often unrecognized because of 
the hegemonic dominance of science and its empirical epistemology that rules out alternative ways of knowing. As the illness and wound of the collective psyche becomes more palpable in our day, there is perhaps nothing more urgent than the need to revive a true psychology or "science of the soul" that is rooted in metaphysics, sacred science, and spiritual principles for the healing of the collective psyche.

This work is written by a Native American who is himself a psychologist and has spent numerous years working in Indian country. The book consists of ten chapters. Chapter 1: Wounding Seeking Wounding: The Psychology of Internalized Oppression describes the wound that has resulted from historical trauma, impacted by the legacy of colonialism and structural racism of the past and present and the factors that perpetuate the traumatization and re-traumatization of indigenous communities. Chapter 2: Overpathologizing Original People, explores the destructive impact of non-indigenous projections onto indigenous peoples, the distinction between traditional knowledge and its modes of healing in contrast with the biomedical or medical model, and how mental health professionals perpetuate historical trauma and the presence of clinical racism in Indian country due to the acculturation of modern Western thought. Chapter 3: The Healing/Therapeutic Circle describes the therapeutic process through its distinct stages consisting of a beginning, middle, and end, the sacred space in which therapy occurs as a ceremony, and the identity of the therapist in this process. Chapter 4: Historical Trauma: Treating the Soul Wound presents vignettes on the healing of the human psyche while simultaneously introducing information on historical trauma and its impact on indigenous peoples. Chapter 5: The Spirit of Alcohol: Treating Addiction presents an alternative view of addiction, framing it as a spiritual disorder, which focuses on the individual developing a new relationship with alcohol to regain balance so that healing can occur. Chapter 6: Diagnosis: Treating Emotional Problems as Living Entities turns to the subject of diagnosis as it is understood in modern Western psychology through the dominance of the biomedical or medical model and how this informs identity, and how this model can be harmful to the individual as it impacts their sense of self; in contrast to the indigenous approach that views the spirit of the illness having visited the person. Yet this spirit of illness does not define who the ill person is, as the relationship with the illness can be transformed; for the illness occurs when someone falls out of balance with the natural order. Chapter 7: "All Conditions Normal": Working with Veterans is a newly added chapter to the second edition of this book that acknowledges the connection between the warrior of the First Peoples and the contemporary veteran, along with the soul wounding that often occurs and how healing takes place. Chapter 8: Community Intervention discusses the necessity that research undertaken on indigenous communities be conducted in a way that appropriately recognizes and honors the uniqueness of their traditional knowledge and healing modalities, which differ from modern Western psychology and its empirical epistemology; this section 
also provides vignettes. Chapter 9: Clinical Supervision again uses vignettes to provide a purview of how supervision can be utilized to provide clinical support and at the same time integrate the spiritual dimension into therapy as it is understood through indigenous epistemology and its healing modalities as outlined in the previous chapters. Chapter 10: Before Completion reminds the reader that the knowledge and therapeutic interventions contained in the text are as old as time and that nothing new per se is discussed in its pages. It points to the universal and timeless wisdom that is neither of the East or West, North or South, belonging to all peoples, and urges the discipline of psychology and field of mental health to take a decolonial turn from the hegemony of modern Western thought.

It needs to be remembered that Native or First Peoples are on the frontline of myriad global issues facing the present day, especially with the environmental crisis. This is so because of their traditional cosmology and metaphysics that establishes their reliance upon, and intimate relationship, with the earth. The environmental crisis greatly compounds the historical trauma and re-traumatization of forced assimilation and genocide, which include political and economic marginalization, loss of land and resources, human rights violations, and racism, among other attacks on their traditional way of life and identity as indigenous peoples.

Colonialism is at the heart of this discussion, as it has had and continues to have such a powerful impact on human identity and one's understanding of themselves and the world. A key shadow element that hangs over the field of modern Western psychology and mental health is that it is not neutral or value-free, nor is it universal as is often assumed. Contemporary psychology has yet to recognize that it is the peculiar creation of the modern Western mindset that has divorced itself from the spiritual dimension. In negating the existence of the human psyche and essentially itself as a psychology or "science of the soul," it fundamentally differs from all other traditional psychologies the world over. Modern psychology is very much a colonizing therapy rather than a decolonizing therapy. It needs to be acknowledged that empiricism is one way of knowing, which is not superior to other diverse epistemologies.

Duran speaks to the fact that many therapists come from or identify with the dominant culture, which itself can be perpetuation of the legacy of colonialism. Because of this, mental health professionals need to be vigilant in not assuming that their worldview is the same as that of the individual they are treating, for this would be an epistemic violation and a form of violence. As the therapeutic relationship is not egalitarian or among equals, the therapist holds the power, which needs to be monitored, as defensiveness can easily turn into the pathologizing of the individual, particularly with indigenous peoples or vulnerable populations. Duran points out that many therapists have assimilated the modern Western worldview and are 
often unaware of how subtle this colonizing process can be. For this reason, it is important for mental health professionals to explore their own cultural history and positions of privilege. Duran points out that through acculturation, Native or First Peoples as mental health professionals may come to identify more with the theories of science and the outlook of the modern world than with their own traditions. This identification could be another "blind spot" that prevents them from detecting the subtlety of mental colonization. He then points out that the opposite could also be true, that an indigenous therapist could hold a great disdain for the practices of modern psychology, which could also hinder the therapeutic process. Duran recommends the need to be flexible with both approaches to the clinical and theoretical outlook of mental health treatment. The best course of treatment depends on the state of mind of the individual seeking services and their acculturation to the modern worldview.

Throughout this book, Duran describes the sacred dimensions of the indigenous ways of knowing and their connection to ceremony. It is critical for the mental health provider to be aware of the physical ambiance in which the therapy is taking place so that it can serve as a healing container. In the therapeutic process, the identity of the mental health professional is important, as it is this identification with the knowledge forms of their own sacred tradition that supports treatment and healing. Duran urges therapists to engage in the timeless inquiry of "Who am I?" for human identity, when it is aligned with our connection with the Spirit, is an essential aspect of the healing container. In this process, the therapist needs to keep in check their understanding of their notions of sickness and healing in order to stay present to what is arising in the "here and now" of the therapeutic encounter. For example, Duran makes a very important point regarding the epidemic of suicide, not only for indigenous communities of the planet, but of the contemporary world itself. He emphasizes that "the idea of wanting to die is literally a misinterpretation of the soul's desire to transform" (p. 100), as "suicide ... [is] a metaphor of transformation" (p. 143), and "suicide is when the soul is asking for transformation" (p. 141).

At the root of the crisis within modern psychology is the Cartesian bifurcation, the dualism between mind and body (and matter) that has plagued the mindset of the contemporary West since the seventeenth century. In this myopic and truncated outlook, the human being becomes separate from reality, and everything becomes objectified, further entrenching the psyche in the subject-object split. This perpetuates the illusion of a separate self, severing the sacredness of relationship, which desacralizes the cosmos and leads to the traumatization of the humanity, sentient beings, and the earth.

Yet across the diverse traditional cultures of the world, this split does not exist, as there is a recognition that the human being is composed of Spirit, soul, and body. Within each of the world's religions_-Judaism, Christianity, Islam, Hinduism, 
Buddhism, Taoism, or the religion of the First Peoples and their shamanic traditionsthere exists a corresponding sacred and integral psychology. To provide the healing of the human psyche requires the spiritual dimension to be restored so that psychology can again be a true "science of the soul." It is through the perennial psychology, in the form of the First Peoples psychology, that a revival of the "science of the soul" is being rehabilitated as a discipline distinct from modern Western psychology (Duran \& Duran, 1995; Katz, 2017; Stewart et al., 2017).

We could not conclude this review without also mentioning the existence of a very unfortunate phenomenon that has emerged within trauma studies. Trauma-informed practices have in many ways become popularized and a profit-generating industry like other money-making endeavors, with countless trauma experts and therapeutic programs proliferating the world. This trend not only muddies the waters but is again a sign of the times.

Duran needs to be commended for his extensive efforts to engage traditional knowledge holders and elders within the Native American community to review the contents of this book prior to its publication to ensure that it authentically represented indigenous modes of knowing and healing modalities. This is a remarkable contribution to the field, along with his other books, and while this work focuses on Native or First Peoples approaches to healing, it is very much a book that will be of interest for all mental health professionals interested in the inclusion of the spiritual dimension in psychology and its application in psychotherapy. The approaches discussed in this work are truly liberating and healing as they encompass the wisdom and respect for the diverse cultures of the world and their religions and spiritual traditions. Duran points out that this work is not a book of techniques to be implemented like a recipe book, but therapeutic strategies that need to be assimilated and meditated upon at the innermost level of the mental health professional. In this way, they can be utilized and employed by the guidance of the Spirit within. We finish this review with a Lakota payer that connects all of humanity and sentient existence to the Great Spirit which is the ultimate source of all medicine, transformation, and healing: "mitakuye oyasin" (We are all related).

\section{References}

Duran, E., \& Duran, B. (1995). Native American postcolonial psychology. State University of New York Press.

Katz, R. (2017). Indigenous healing psychology: Honoring the wisdom of the first peoples. Healing Arts Press.

Stewart, S. L., Moodley, R., \& Hyatt, A. (Eds.). (2017). Indigenous cultures and mental health counselling: Four directions for integration with counselling psychology. Routledge. 